\title{
Vehicular Networking: ITS-G5 vs 5G Performance Evaluation using Road Weather Information
}

\author{
Muhammad Naeem Tahir \\ Arctic Space Center, Finnish \\ Meteorological Intitute (FMI-ARC) \\ Helsinki, Finland \\ naeem.tahir@,fmi.fi
}

\author{
Timo Sukuvaara \\ Arctic Space Center, Finnish \\ Meteorological Intitute (FMI-ARC) \\ Helsinki, Finland \\ timo.sukuvaara@fmi.fi
}

\author{
Marcos Katz \\ Centre for Wireless Communications \\ (CWC) - Networks \& Systems \\ University of Oulu, Finland \\ marcos.katz@oulu.fi
}

\begin{abstract}
Safety of transport systems on roads has become the crucial developmental concern within the road infrastructure. The prevailing climatic situation plays a crucial role on transport safety and it is a key cause behind weather hazards in traffic, leading to accidents in northern regions of Europe and America. In this article, we present the test results of pilot road weather related services on a set of Vehicle-toInfrastructure (V2I) communication scenarios by using ITS-G5 (IEEE 802.11p) and 5G test network with TCP and UDP. TCP/UDP are the transport layer connection orientated (TCP) and connectionless (UDP) IP protocols that define how information on internet can be exchanged. The Finnish Meteorological Institute (FMI) has a test track equipped with both Road Weather Stations (RWS) and a 5G test network (5GTN). These networks have the capability of supporting ITSenabled road weather services in very realistic situations. We evaluated the performance of ITS-G5 and 5GTN with transport layer TCP and UDP. The performance is investigated by considering average throughput and lost packets. The analysis of transport layer protocols provides us a deep insight of vehicular networking and performance degradation factors. Even though IEEE 802.11p is specifically designed for vehicular communication, the soon-to-be deployed 5G technology would be able to offer the reliability to satisfy functional safety requirements with almost $80 \%$ of TCP data traffic. The evaluation indicated that the state-of-the-art 5G technology would be a better substitute to fulfill majority of the required features for vehicular applications, including latency, throughput and others.
\end{abstract}

Keywords-Vehicular Networking, IEEE-802.11p, 5G test network, ITS, RWS

\section{INTRODUCTION}

Intelligent transport system (ITS) refers to the trafficenvironment communication entity comprised of sensors, controllers and other communication devices improving the efficiency, mobility and safety of the transportation system. The main objectives of an ITS system is to reduce road congestion, improve road safety, traffic efficiency and energy efficiency, as well as to reduce carbon dioxide (CO2) emission. The deployment of advanced technologies in ITS focuses on four fundamental principles namely safety, sustainability, integration and responsiveness. These unconventional technologies will be supported by the transportation systems supporting the efficient use of infrastructures to manage, regulate and control the traffic [1].

Consistent efforts and research work are being done by numerous regulatory authorities to facilitate ITS application scenarios i.e., vehicle-to-infrastructure (V2I) and vehicle-tovehicle $(\mathrm{V} 2 \mathrm{~V})$. Advanced communication systems are crucial support for vehicular communication in real-time ITS applications. The most significant development of radio communication technology for ITS applications has been developed e.g., advance safety features for road transport applications by getting road weather and accident information.

Road weather information adds travel convenience and safety in V2V and V2I scenarios. Meanwhile, considerable benefits are also expected by road users in terms of improved traffic management, fuel savings, congestion avoidance, safety and comfort by using V2V and V2I communication systems $[1,2]$. There are few radio technologies available for vehicular networking that can improve and enhance the operation and performance of V2I and V2V systems. The VANET requires a seamless radio link between V2I and V2V whereas, IEEE $802.11 \mathrm{p}$ has been specially designed for this purpose. Meanwhile, the $3^{\text {rd }}$-Generation Partnership Project (3GPP) cellular communication systems by default offers extended coverage, the missing feature in VANET.

Figure 1 depicts a V2V and V2I communication system using IEEE 802.11p and 5G utilizing road weather data with IOT cloud. VANET (IEEE 802.11p) and cellular technology $(4 \mathrm{G} / 5 \mathrm{G})$ standards are currently the most significant standards for vehicular communication within the context of our pilot measurements and performance evaluation for use cases dealing with ITS safety [3].

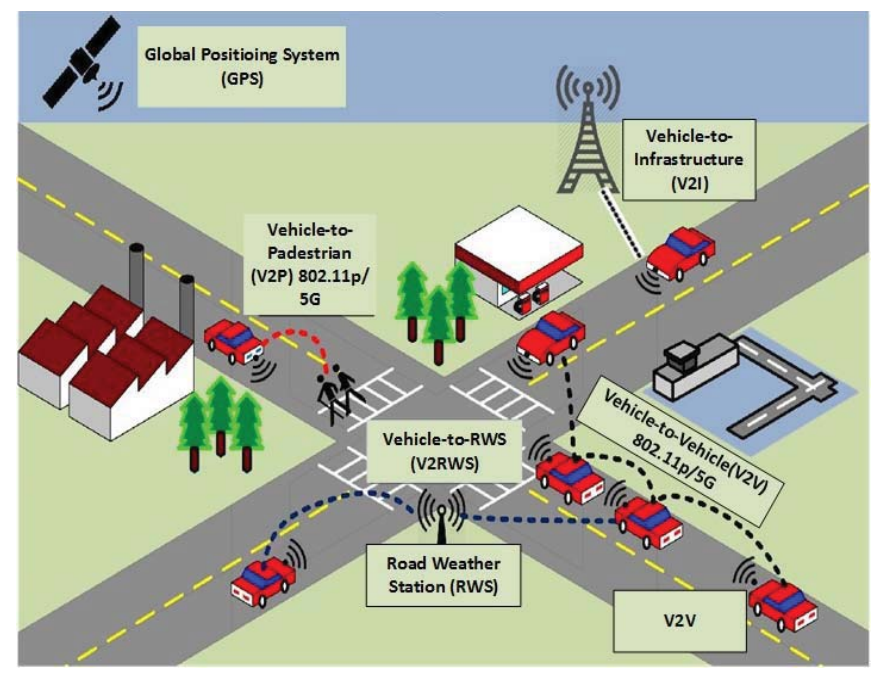

Fig. 1. Intelligent Transport System using VANET (IEEE 802.11p) and Cellular Network (5G) (4)

In $\mathrm{V} 2 \mathrm{~V}$ and $\mathrm{V} 2 \mathrm{I}$ communications, two transport layer protocols are used for end-to-end data transfer namely User Datagram Protocol (UDP) and Transmission Control Protocol (TCP). TCP offers connection-oriented communication that provides the network with handshake, error checking, acknowledgements, flow control, etc. On the other hand, UDP relies on retransmission without any such mechanisms. The TCP provides reliability at the cost of transmission delay, in contrast of UDP.

For the development of ITS time-critical (emergency vehicle warning system and automated road speed 
enforcement, etc.) applications, we need to recognize and understand the expected performance of the underlying VANET (IEEE 802.11p) and cellular networks (5G) considering TCP/UDP. To understand the performance analysis of vehicular networks, we conducted pilot measurements on a $1.7 \mathrm{~km}$ long test track owned by Finnish Meteorological Institute (FMI) in Sodankylä, Finland. We have analyzed the network performance in V2I scenario using TCP/UDP by investigating the average throughput and lost packets (\%). V2I communication was performed from a moving vehicle to the Road Weather Station (RWS) using different wireless standards (IEEE 802.11p and 5GTN). To the best of our understanding, performance evaluation at transport layer in vehicular networking has not been studied in detail before [5].

This article provides an overview of IEEE 802.11p and 5GTN considering transport layer (TCP and UDP) protocols. The rest of the article is organized as follows. Section 2 presents the general overview of vehicular networking, Section 3 presents the ITS and road weather information system, Section 4 illustrates the ITS radio technologies (IEEE $802.11 \mathrm{p} \&$ \& GTN) and protocol architecture, Section 5 provides the pilot measurements setup in V2I scenario using TCP \& UDP and section 6 illustrates the performance evaluation of results respectively. Section 7 concludes the article.

\section{ITS \& ROAD WEATHER SERVICES}

Nowadays, ITS research is striving for operative devices, systems and services to get correct location data together with all constraints associated with its safety limit with respect of other traffic entities. To be able to get the required road safety related information, the road weather services play a crucial role of ITS, as depicted in Fig. 2. They exploit road traffic data by permitting more precise and accurate instant service generation directly to different traffic and transport actors. For traffic safety, the next generation of applications and services aims to include the combination of road weather and wireless communication link together (VANET \& cellular network) [6]. This combination "Next Generation Application" can provide the advanced specifications that forecast, assess and address the climatic impacts on roads, travelers and vehicles. The motive behind this application is to study and perform research to develop greater specificity related to the weather prediction and its impact on the roadways that can call for various strategies to mitigate the effects. With the help of such designed applications, real-time information is effectively used to increase the situational awareness and make the roadway level of service more efficient. It can also optimize the use of resources and equipment's to inform run-time traffic alerts and increase the ability to respond quickly to the adverse effects of the weather and roadway surface conditions in order to eliminate the weather-related delays and car crashes [7].

Harsh winters in the northern countries of Europe are one of the key causes of road traffic problems. The main goal of ITS research unit at FMI is to design and develop application services to tackle the road traffic problems. These services support normal users as well as ITS business development by providing the road safety provision through the vehicular networking system. The services consist of accurate and reliable weather observations, related warnings and weather forecasts. Moreover, they provide predictions of air quality, road surface condition information, warnings of poor sections, safety margin warnings, fog vision recognition, improved driver visibility and behavior recognition. To increase the traffic safety, IEEE 802.11 p was introduced in 2012 to satisfy the requirement of uninterrupted communication between V2I and V2V. However, the communication system of the vehicular safety messaging cannot tolerate long connection placement delays associated with centralized safety systems. For this purpose, the cellular communication $(4 \mathrm{G} / 5 \mathrm{G})$ is really helpful, complementing the missing feature of wide range coverage. The $4 \mathrm{G} / 5 \mathrm{G}$ networking has also a vision of providing the vehicular communication-type of operation with high bandwidth and ultra-low network delays. Radio technology together with road weather service applications can improve the safety application efficient as well as the traffic flow [6, 7].

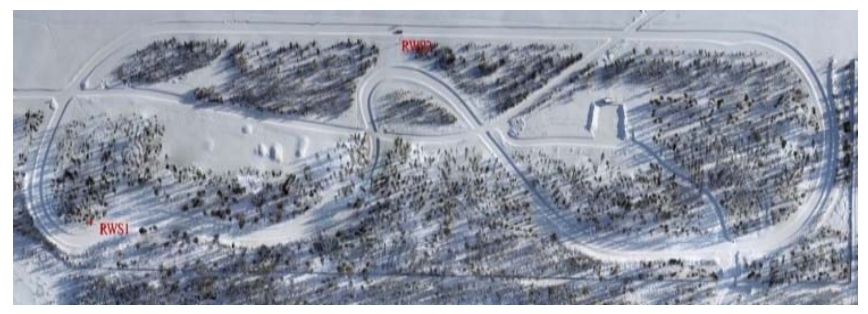

Fig. 2. Aeriel view of road weather stations proving road weather information for ITS application

\section{ITS RADIO TECHNOLOGIES AND RADIO PROTOCOL ARCHITECTURE}

\section{A. IEEE $802.11 p$}

IEEE 802.11p is an adapted version of the IEEE 802.11 Wi-Fi standard was developed to meet the lacking required features in the Wireless Access in Vehicular Environments (WAVE) to support ITS applications. Fig. 3 presents the WAVE protocols architecture with IEEE 802.11p and IEEE 1609. IEEE 1609 is a higher layer standard based on the IEEE 802.11p. IEEE 802.11(p)/1609 standard supports the suit of TCP/UDP/IP protocol with WAVE-mode short message protocol (WSMP). IEEE 1609 is a WAVE Family of Standards to enhance the security of the wireless communication between V2V and V2I. This IEEE 802.11p standard includes a feature to exchange data between high speed vehicles and roadside-infrastructure so called V2X communication [8]. IEEE 802.11p is also the base for a European standard for vehicular communication known as ETSI ITS-G5. IEEE 802.11p improves the communication on the physical layer in terms of developed WAVE (wireless access in vehicular environments and ETSI ITS-G5 standards) [9]. The respective technologies have proven to meet all the requirements in vehicular communication systems as the extensive field operational trials have been performed in various countries of Europe and US as well. Meanwhile industries have developed transceiver chipsets that permits mass production for deployment at large scale. The vehicular communication with the licensed ITS band (5.9 $\mathrm{GHz}$ with $10 \mathrm{MHz}$ of bandwidth) has been allocated in both US Direct Short Range Communication (DSRC) and Europe (C-ITS) $[8,9]$. More development is vital for the climate and traffic conditions updates. For real-time weather alerts and road weather information, roadside infrastructures including 
sensors and cameras are used to collect the expected data about the environmental and traffic conditions. The synergistic integration of the vehicular sensing devices and communication capabilities calls for ITS. The $802.11 \mathrm{p}$ standard has the ability to provide the best available features of the vehicular communication approach. 802.11p has the ability to operate in a rapid fluctuating environment to exchange vehicular data. It provides various V2X application requirements with the most advanced specifications as well as it can also assist to reduce the number of collisions on the roads. Conversely, the $802.11 \mathrm{p}$ is inadequate to curtail the load of vehicular communication load. This is primarily due to the high message frequency requirements of safety applications in a shared and limited wireless spectrum [10]. IEEE $802.11 \mathrm{p}$ also deals with the congestion control technique such as contention window adaptations, message frequency and transmit power at the medium access control (MAC) layer and allow an uncoordinated and fully distributed access to the wireless channel with no compulsion of resource allocation procedure. The physical layer (PHY) is used with eight possible arrangements of Modulation and Coding Scheme (MCS) methods. Depending on the application specific adopted MCS, the $802.11 \mathrm{p}$ data rate ranges between 3 and 54 Mbps.

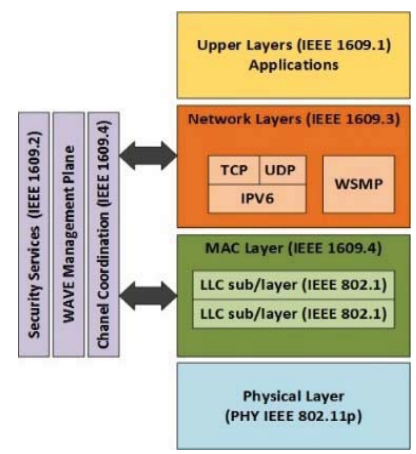

Fig. 3. WAVE protocols architecture: IEEE 802.11p and IEEE 1609

\section{B. $5 G$ Wireless network}

The $5 \mathrm{G}$ is the $5^{\text {th }}$ generation cellular technology and $3^{\text {rd }}$ Generation Partnership Project (3GPP) submitted the $5^{\text {th }}$ Generation New Radio (5G-NR) to International Telecommunication Union (ITU). 5G-NR is the Radio Access Technology (RAT) developed by 3GPP with two frequency ranges; Frequency Range 1 works under $6 \mathrm{GHz}$, and Frequency Range 2 that includes bands over $24 \mathrm{GHz}$ and with the extreme high frequency range beyond $50 \mathrm{GHz}$. This $5 \mathrm{G}$ standard follows $2 \mathrm{G}, 3 \mathrm{G}$ and $4 \mathrm{G}$ and their respective associated technologies. This $5 \mathrm{G}$ network will facilitate ITS infrastructure to lead the establishment of intelligent cities with the deployment of ultra-low latency networks for the distribution of real-time data between both vehicles and transport infrastructure. One of the ITS challenges is the problem in vehicular networking to cope with the high speed vehicles, where the users are not able to detect other vehicles for vehicular communication. Moreover, the pedestrians could have enough time to respond to the possible threats that may be encountered. The deployment of $5 \mathrm{G}$ wireless communication systems allows vehicles to get alerts from traffic management system. It also enables to adjust the patterns of traffic and provides the optimal routes to the vehicles in run-time, specifically the emergency services. 5G network infrastructures promises a much easier time for upgrading the technologies in the future by combining the connectivity, shared standards, wireless communication and Internet of Things (IoT) [11]. 5G network would able to offer ultra-low latency of $1 \mathrm{~ms}$ at up to the speed of $500 \mathrm{~km} / \mathrm{h}(310$ $\mathrm{mi} / \mathrm{h})$. 5G network can play a vital role in automotive connectivity due to its ultra-low latency. Future cellular technologies will provide smoother upgrade paths that will aid to provide up-to-date and stronger services including security to protect both the ITSs and the wireless networks themselves [12].

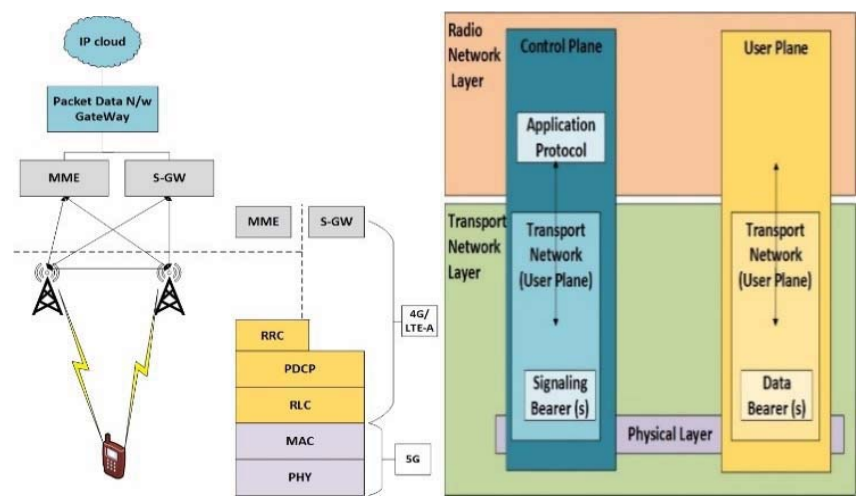

Fig. 4. 5G Framework \& Protocol Architecture

Fig. 4 illustrates the $5 \mathrm{G}$ framework and protocol architecture comprising of two main parts, named as core access and radio access network. The core access network is also recognized as a NAS (Non-access stratum) that participates in the necessary mechanisms to establish a connection among cell towers and external IP networks. In $5 \mathrm{G}$ framework, each element perfrorms as a separate server for the execution of specific set of functions [11, 12]. Fig. 4 presents the radio protocol architecture for $5 \mathrm{G}$ that can be divided into user plane and control plane architecture. The user plane side of the $5 \mathrm{G}$ protocol architecture has the application that creates data packets and further managed by IP protocols such as UDP and TCP. The control plane has the radio resource control (RRC) protocol that executes the signaling data and also transfers data between the cell phone and base station. In both (user plane and control plane) cases, the data is managed by the PDCP (packet data convergence protocol), the RLC (radio link control) protocol and the MAC (medium access control protocol), before sending the data to the physical layer for transmission.

\section{PILOT MEASUREMENT SCENARIO FOR 802.11P AND 5G TEST NETWORK}

This section focuses on V2I data sharing in the surrounding vehicles and infrastructure. RWS offers real-time up-to-date weather information, friction data of roads collected by different vehicles with the help of different sensors i.e., Teconer [13]. RWS gathers the observation data from the vehicles (V2I) passing nearby, to utilize the information for ITS services and applications. RWS provides the collective (vehicles and RWS data) information to the vehicles (V2I). The vehicles following another vehicle or passing nearby would also be able to make an ad hoc network and that will extend the range of RWS [13]. By using this ad hoc network, the RWS would be able to forward this information to other vehicles $(\mathrm{V} 2 \mathrm{~V})$, hence propagating the RWS data by extending the RWS range. Traffic safety is a 
crucial issue in this approach and this V2I scenario provides a solution by gathering friction data from the sensors in vehicles and getting real time weather updates from RWS to avoid accidents or getting weather alerts to the vehicles and RWS.

TABLE I. $\quad$ 802.11P PARAMETER SETTINGS

\begin{tabular}{|l|l|}
\hline \multicolumn{1}{|c|}{ Parameters Description } & \multicolumn{1}{|c|}{ Technical Settings } \\
\hline Transmission Power & -10 to $+23 \mathrm{dBm}$ \\
\hline Frequency Band & $5.9 \mathrm{GHz}$ \\
\hline Modulation Technique & $\mathrm{BPSK}, \quad$ QPSK, \\
& $16 \mathrm{QAM}, 64 \mathrm{QAM}$ \\
\hline $\begin{array}{l}\text { Maximum Transmission } \\
\text { Rate }\end{array}$ & $27 / 54 \mathrm{Mbps}$ \\
\hline Data Traffic & Bi-directional \\
\hline Bandwidth & $10 \mathrm{MHz}$ \\
\hline Supply Voltage & $3.3 \mathrm{~V}$ \\
\hline Temperature & $-40^{\circ} \mathrm{C}$ to $+85^{\circ} \mathrm{C}$ \\
\hline Maximum Range & $1000 \mathrm{~m}$ \\
\hline
\end{tabular}

TABLE II. 5G Test Network Parameter Settings

\begin{tabular}{|l|l|}
\hline \multicolumn{1}{|c|}{ Parameters Description } & \multicolumn{1}{|c|}{ Technical Settings } \\
\hline Transmission Power & $41.8 \mathrm{dBm}$ \\
\hline Frequency Band & $2.3 \mathrm{GHz}$ \\
\hline \multirow{2}{*}{ Modulation Technique } & $64 \mathrm{QAM}$, \\
& $16 \mathrm{QAM}$ \\
\hline Data Rate (each user) & $10 \mathrm{Mbps}$ \\
\hline Data Traffic & Bi-directional \\
\hline Bandwidth & $40 \mathrm{MHz}$ \\
\hline Supply Voltage & $230 \mathrm{volts}$ \\
\hline Antenna gain (Tx and Rx) & $19 \mathrm{dBi}$ \\
\hline Maximum Range & $1000-1700 \mathrm{~m}$ \\
\hline
\end{tabular}

For pilot measurements, we used road weather stations and $5 \mathrm{G}$ test network with one vehicle for the V2I scenario. The pilot measurements were performed by driving a vehicle in the $1.7 \mathrm{~km}$ closed-loop test track equipped with two RWS and a $5 \mathrm{G}$ test network, performing as a V2I counterpart. Fig. 5 illustrates the $5 \mathrm{G}$ test network base station and RWS on FMI test track. The vehicles and RWS networking were piloted with Cohda radio MK5-transceivers, providing compatibility to the IEEE WAVE standard $802.11 \mathrm{p}$. The parameter settings for $802.11 \mathrm{p}$ and $5 \mathrm{G}$ test network pilot measurements are mentioned in Table 1 and Table 2. For user interface (UI) in vehicles, we used SUNIT F-series vehicle PC, tablets (android) can also be used as an alternative solution.

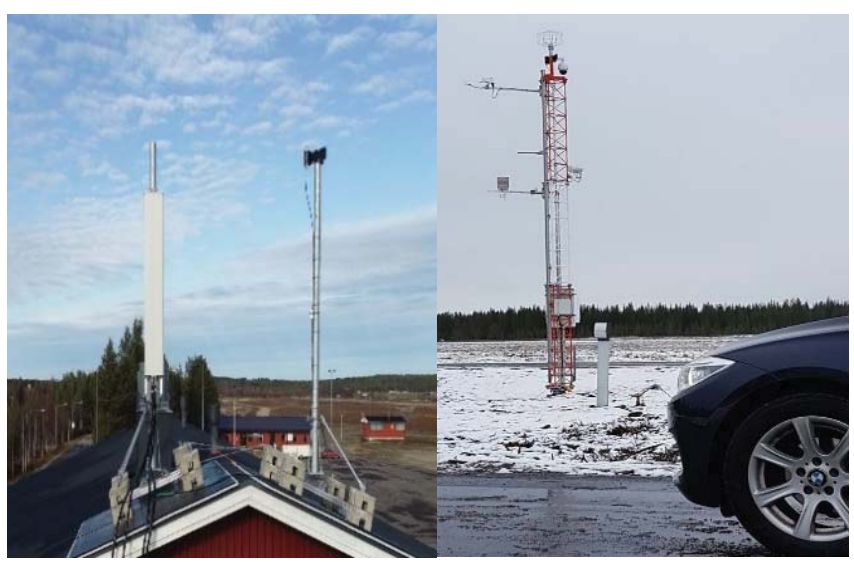

Fig. 5. 5G Test Network and RWS on test track

In V2I vehicular networking, the data is archived solely from the external friction measurement sensors mounted on the road borders and vehicles. In these measurements, we used RCM411 road condition observing devices with road state, road friction and road temperature sensors i.e., RTS411/RTS411SA.

While driving on the test track, the vehicle collects the updated RWS data in V2I communication mode and RWS distributed the up-dated weather information to the vehicles (V2I), while passing nearby. Moreover, the nearby vehicles exchange the run-time collected friction information and weather alerts collected from the RWS. This pilot vehicular networking offers almost the continuous networking during the tests on our test track. Fig. 6 and Fig. 7 depict the IEEE 802.11p and 5G test network connectivity for our pilot measurements in the V2I scenario. The yellow spots indicate the locations where the TCP and UDP packets were captured in Vehicle to RWS (V2I) scenario.

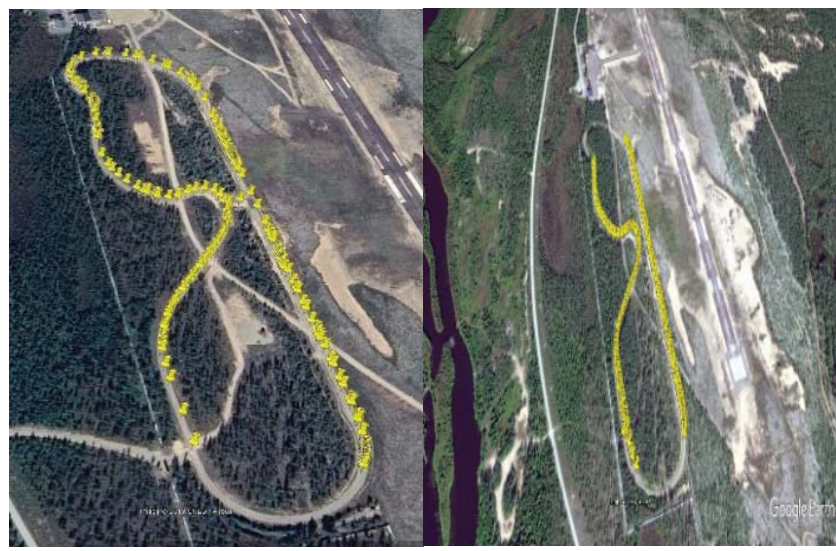

Fig. 6. IEEE-802.11p, TCP \& UDP packet capture V2I Scenario

The data exchange between vehicle and road weather station (V2I) was performed in with 13 drives in a closed loop of test track, as presented in Figs. 6 and 7.

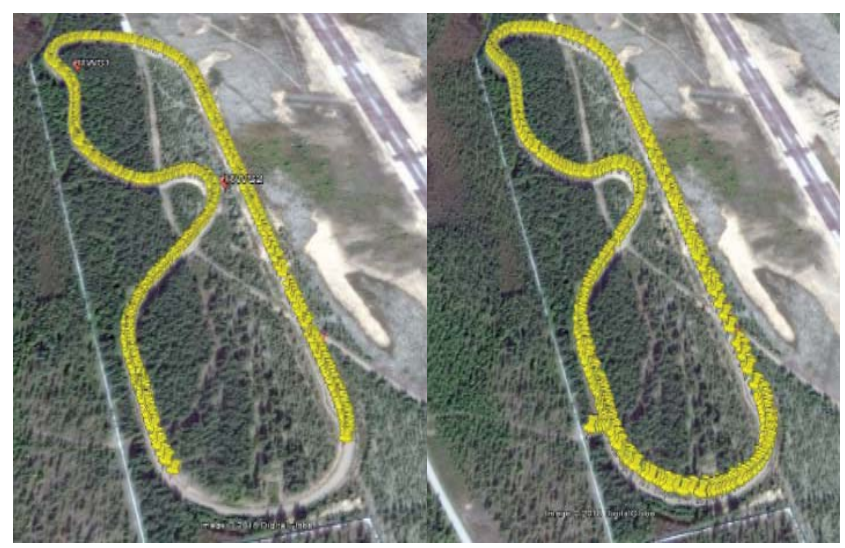

Fig. 7. 5G Test Network, TCP \& UDP packet capture V2I Scenario

\section{PERFORMANCE ANALYSIS OF IEEE 802.11P AND 5G TEST NETWORK IN V2I SCNARIO}

\section{A. IEEE 802.11p (TCP) vs 5GTN (TCP)}

In this section we analyze the performance of IEEE $802.11 p$ and $5 \mathrm{G}$ test network in V2I pilot scenarios using transport layer protocols TCP and UDP. The performance evaluation is performed by considering the average throughput, data rate and lost packets (\%) and it is presented in Table III. In this TCP analysis (V2I), 5G test network is dominating in terms of average throughput (TCP). The low average throughput of $802.11 \mathrm{p}$ is mainly caused by the packet loss and the network latency (due to windowing). In Table III, 
we can also see that the average throughput suffers significantly in $802.11 \mathrm{p}$, as compared to $5 \mathrm{G}$ test network. Similarly, the data rate also get affected due to the large amount of data that $802.11 \mathrm{p}$ could not been able to assure the requirements for transmission information of time sensitivity. The $802.11 \mathrm{p}$ and 5GTN have a bandwidth of $10 \mathrm{MHz}$ and $40 \mathrm{MHz}$ respectively that also effects the data rate of $802.11 \mathrm{p}$.

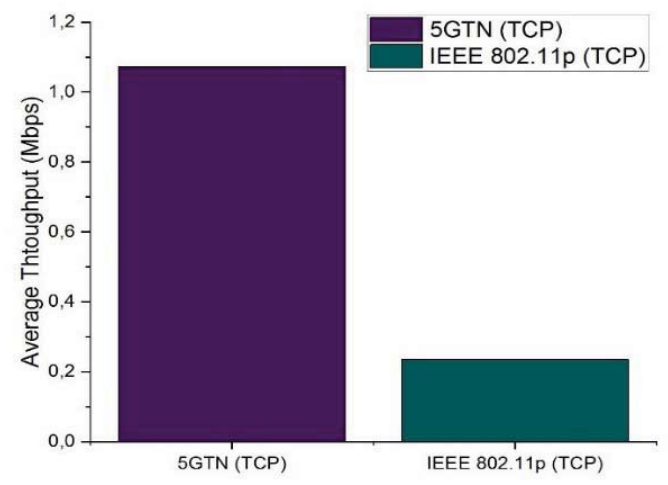

Fig. 8. 5GTN (TCP) Vs IEEE 802.11p (TCP)

The packet loss in Table III also clearly illustrates that 5GTN has less packet loss in contrast of $802.11 \mathrm{p}$, due to embedded system in our RWS. The larger packet size (1431 bytes) in $802.11 \mathrm{p}$ increases the window time, resulting in increased network latency, and ultimately the network suffers from high packet loss affecting the throughput. The average throughput of $802.11 \mathrm{p}$ and 5GTN with TCP is illustrated in Fig.8, clearly showing the superiority of 5GTN in our V2I pilot measurements with TCP.

TABLE III. 5G TEST NETWORK: V2I

\begin{tabular}{|l|l|l|}
\hline \multicolumn{1}{|c|}{ Analysis Factors } & \multicolumn{1}{|c|}{ 5GTN (TCP) } & \multicolumn{1}{|c|}{$\mathbf{8 0 2 . 1 1 p}$ (TCP) } \\
\hline Data Rate (Mbps) & 1.12 & 0.394 \\
\hline $\begin{array}{l}\text { Average Throughput } \\
\text { (Mbps) }\end{array}$ & 1.05 & 0.220 \\
\hline Packet Loss (\%) & 22 & 28 \\
\hline
\end{tabular}

\section{B. IEEE $802.11 p(U D P)$ vs $5 G T N(U D P)$}

In this section, we compare the IEEE $802.11 \mathrm{p}$ and $5 \mathrm{GTN}$ by using transport layer UDP in the V2I scenario. Table IV presents the packet loss in 5GTN and 802.11p. One of the reasons for packet loss is the initial connection setup with RWS/base station and the other reason is a moving vehicle on a test track might face locations with poor wireless link quality between vehicles and RWS. The possibility of packet loss in UDP is always high due to continue transmission of packets and this aggressive UDP data flow consumes most of the network bandwidth therefore, affecting the data rate, throughput and packet loss. The 5GTN and 802.11p bandwidth $40 \mathrm{MHz}$ and $10 \mathrm{MHz}$ respectively, that ultimately affects the average throughput and data rate.

TABLE IV. IEEE 802.11P: V2I

\begin{tabular}{|l|l|l|}
\hline \multicolumn{1}{|c|}{ Analysis Factors } & \multicolumn{1}{|c|}{ 5GTN (UDP) } & \multicolumn{1}{c|}{ 802.11p (UDP) } \\
\hline Data Rate (Mbps) & 4.96 & 2.21 \\
\hline $\begin{array}{l}\text { Average Throughput } \\
\text { (Mbps) }\end{array}$ & 4.62 & 2.19 \\
\hline Packet Loss (\%) & 39 & 36 \\
\hline
\end{tabular}

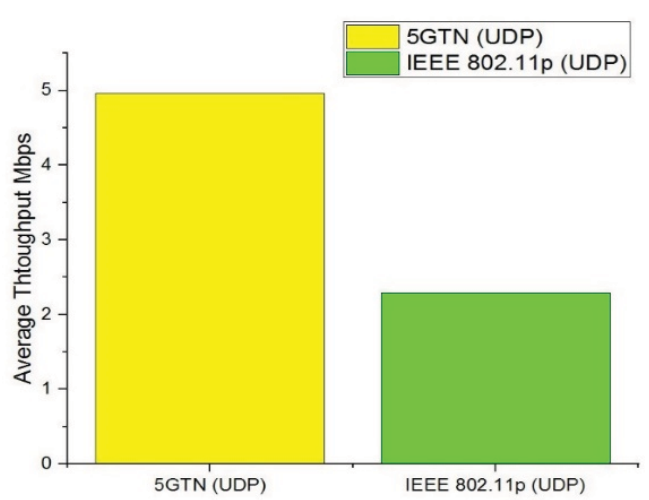

Fig. 9. 5GTN (UDP) Vs IEEE 802.11p (UDP)

\section{CONCLUSION}

In this paper, we have evaluated the performance of $5 \mathrm{GTN}$ and ITS-G5 in realistic V2I scenarios with the help of iperf, Wireshark and python programs. The packet loss rate in our vehicular pilot measurements was quite normal with TCP and UDP due to wireless link. According to our results, 5GTN offers more network coverage as compared to $802.11 \mathrm{p}$ with both TCP and UDP in the V2I scenario. By using TCP in the pilot measurements, we noticed that the both (ITS-G5 and 5GTN) networks work reliably at the cost of network latency that affects the data rate and average throughput. Basically, IEEE $802.11 p$ is a narrowband communication standard that works well with UDP by sending continuous transmission packets. The pilot measurement results show the UDP as a best protocol with 5GTN due to its low transmission delay and network latency. Meanwhile TCP is very sensitive in vehicular environments due to network latency and packet loss that limit its throughput. With the launching of $5 \mathrm{G}$ networks for $\mathrm{C}-\mathrm{V} 2 \mathrm{X}$, TCP would be able to provide such features to deal with delay and latency that mitigates the jitter and latency to provide errorless vehicular networking. The aim of this article was to investigate the performance of vehicular communication technologies $802.11 \mathrm{p}$ and $5 \mathrm{G}$ under different network configurations and in a realistic operating environment. A robust solution could be a development of tailored cost-effective heterogeneous (802.11p and 5G) vehicular networking in the near future. Lastly, we conclude the discussion with an observation that $5 \mathrm{G}$ offers some benefits over IEEE $802.11 \mathrm{p}$ in numerous aspects, but still has time till its launch for vehicular networking and to have full compatibility with $\mathrm{C}-\mathrm{V} 2 \mathrm{X}$. As future work, we have a plan to measure the performance of IEEE 802.11p and 5G using drones in a large-scale vehicle use cases

\section{REFERENCES}

[1] Tahir, M. N., Maenpaa, K., \& Sukuvaara, T. (2019, March). Evolving Wireless Vehicular Communication System level comparison and analysis of 802, 11 p, 4G 5G. In 2019 2nd International Conference on Communication, Computing and Digital systems (C-CODE) (pp. 4852). IEEE.

[2] Wang, G., Chen, X., Zhang, F., Wang, Y., \& Zhang, D. (2019, May). Experience: Understanding long-term evolving patterns of shared electric vehicle networks. In The 25th Annual International Conference on Mobile Computing and Networking (pp. 1-12). ACM.

[3] Ullah, H., Nair, N. G., Moore, A., Nugent, C., Muschamp, P., \& Cuevas, M. (2019). 5G Communication: An Overview of Vehicle-toEverything, Drones, and Healthcare Use-Cases. IEEE Access, 7, 37251-37268.

[4] Javed, M.A.; Ben Hamida, E.; Znaidi, W. Security in Intelligent Transport Systems for Smart Cities: From Theory to Practice. Sensors $2016,16,879$. 
[5] Tahir, M. N., Maenpaa, K., \& Sukuvaara, T. (2019, April). Analysis of SafeCOP Features in V2I and V2V Communication. In 2019 IEEE 89th Vehicular Technology Conference (VTC2019-Spring) (pp. 1-6). IEEE.

[6] Sukuvaara, Timo, Kari Mäenpää, and Riika Ylitalo. "Vehicularnetworking-and road-weather-related research in Sodankylä." Geoscientific Instrumentation, Methods and Data Systems 5.2 (2016): 513-520.

[7] Dey, K. C., Mishra, A., \& Chowdhury, M. (2014). Potential of intelligent transportation systems in mitigating adverse weather impacts on road mobility: A review. IEEE Transactions on Intelligent Transportation Systems, 16(3), 1107-1119.

[8] Klapez, M., Grazia, C. A., Rold, L., \& Casoni, M. (2019, July). IEEE $802.11 \mathrm{p}$ under congestion in an Infrastructure-to-Vehicle communication approach. In 2019 AEIT International Conference of Electrical and Electronic Technologies for Automotive (AEIT AUTOMOTIVE) (pp. 1-6). IEEE
[9] Altay, C., Atli, A. V., Civanlar, S., \& Ercan, A. O. (2019). U.S. Patent Application No. 15/945,941.

[10] Ge, X. (2019). Ultra-Reliable Low-Latency Communications in Autonomous Vehicular Networks. IEEE Transactions on Vehicular Technology, 68(5), 5005-5016.

[11] Wang, Min, et al. "Comparison of LTE and DSRC-based connectivity for intelligent transportation systems." Vehicular Technology Conference (VTC Spring), 2017 IEEE 85th. IEEE, 2017.

[12] Vinel, Alexey, et al. "Emerging technology for 5G-enabled vehicular networks." IEEE Wireless Communications 24.6 (2017): 12-12.

[13] Sukuvaara, Timo. (2015). Development, implementation and evaluation of an architecture for vehicle-to-vehicle and vehicle-toinfrastructure networking. 10.13140/RG.2.1.3004.1045. 